\title{
Research Significance of Wing and Vein among Insecta
}

\author{
Bingbing Cui \& Guangchun Liu \\ Shenyang University, No. 21 Wanghua South Street, Dadong District \\ Shenyang 110044, China \\ Tel: 86-24-2324-3799Ｅ-mail: bingb-ndb@163.com
}

\begin{abstract}
Insects are the only flying animals among invertebrates, but also the earliest animals with wings in animal kingdom. The presence of wings not only expands the insects' activity and distribution scope, but also enhances their speed, which brings superiority and competence in variety of aspects such as foraging, calling, finding places for spawning, winters and summers, avoiding predators and so on. Wing is an important condition for insecta to become the most prosperous biological community. Among various insects, wings experienced a wide range of variation, so the characteristics of wings have become an important basis for the study of insect classification and evolution.
\end{abstract}

Keywords: Insect, Wing, Vein

Insect is one of the largest animal groups in animal kingdom, individual number, biomass, specie number and gene number all play an important role in biological diversity. The relationship between insect and human is complicated and close, some insects caused great tragedy to human being, and some provide a plenty of resources. Insecta is the largest class in animal kingdom. During the process of development and growth, insects usually become sexually mature through a series of internal and external morphological variation. Insects are the earliest animal group with flying ability in animal kingdom, but also the only animal group with wings in invertebrates. Flight ability is one of the important factors for insect groups to be prosperous. Since Lorenz Oken brought forward the issue of the origin of insect wings in 1811, two centuries have been away. Although some theories have been put forward by several scholars, none of them was a conclusive theory so far.

\section{Basic structure of insect wings (Cai, 2001)}

Wings were seemingly triangular in general, and thus have 3 margins and 3 angles. When it was placed flat, the margin close to the side of the head called costal margin, close to the side of tail called posterior margin or inner margin, and between costal margin and posterior margin called outer margin. The angel in wing base called humeral angle, the angel between costal margin and outer margin called apical angle, and between outer margin and inner margin called anal angle. Insects with wings of new types meet with the folding and flying of wings, therefore, three flexion creases of wings divided wing surface into four regions. Triangular region with axillaries in wing base called axillary region; the fold outside axillary region called basal fold; Vannal fold and jugal fold extending from the external angle of axillary region divided other parts excluding axillary region into 3 regions, namely regigium, vallal region and jugal region or neala. Remigium was generally well developed, but vallal region of hind wings in orthopteran was well-developed. Flies of Diptera had 1-2 membranous flaps in the posterior margin base of forewings, namely aileron. Some insects had a deep stain in both sides of wing costal margins, namely pterostigma.

\section{Insect wing vein types and neuration}

\subsection{The concept of veins}

Veins are stripes distributed vertically and horizontally in the two thin layers of wings, formed by the thickening of trachea parts, and play a supportive role for wing surface.

\subsection{Types of veins}

\subsubsection{Longitudinal and cross vein}

Longitudinal vein is the vein sending out from wing base and extending to wing margin, and starting at the branch of trachea trunk in foot trachea, main body of wings. Cross vein is the short vein ranked between longitudinal veins, and acts as supporting wing surface. 


\subsubsection{Convex vein and concave vein}

The wing surface in most insects is not absolutely flat, especially in more primitive species. Wing surface in general looks like gloss and irregular folding fan, concave and convex alternate in longitudinal veins, and seen from the back, vein located in the top of the fold call convex vein.

2.2.3 Accessory vein, intercalary vein and serial veins

Accessory vein acts as the branch of longitudinal veins, and its nomenclature principle is the shorthand of original vein followed by lower case English letters, a, b, c etc. in turn. Intercalary veins are several inserted thin longitudinal veins between two adjacent longitudinal veins, and thus they are also called intercalary veins or internal veins. Serial vein is a vein formed by the connection with the subsections of two or more veins.

\subsection{Venation}

Venation means the distribution form of veins in the wing surface. There are some differences among different types of veins, but veins of the same type are relatively stable; accordingly, venation is one of the most important traits in insect phylogenetic development.

\section{Wing joint and movement and wing chain}

\subsection{Wing joint}

Articulation is the material basis for wing activities, and the production of articulation is the prerequisite condition for the transformation from gliding function to flight operation. Insect articulation includes several groups of bone plates in membranous area of wing base, which are collectively called pteralia.

3.1.1 Humeral plate is a small bone piece at the base of wing outer margin, and the movement joint for costal margin vein base. Humeral plate in the insects of Odonata is well-developed.

3.1.2 Axillary plates are a group of (3-4) bone plates in the axillary region. The first axillary piece plate is internally connected to medalaria and backplane lateral margin; the front part outside is connected to postcosta, and the back part is connected to the second axillary plate. The second axillary plate is internally connected to the first axillary plate; The front part outside the plate is connected to radial vein in the top, the middle part is connected to medium plates, and the back part is connected to the third axillary plate; In the ventral surface of the plate, there is a little depression, the sudden wing of side panel is tightly connected to the depression; obviously, the second axillary plate is the most important fulcrum for wing movement. Posterior margin of the third axillary plate wrapped upwardly, inner anterior angle is branch connected to the second axillary plate, and inner posterior angle is branch connected to the sudden wing of rear back, costal margin is internally connected to inner medium plates, and outer angel supports anal vein base; the plate is the important bone piece to make wing rotate and fold. The fourth axillary plate exists in a few insects.

3.1.3 Median plates are the two weaker ossification bone plates; inner median plate is internally connected to the second axillary plate, backwardly connected to the third axillary plate, and outwardly branch connected to outer median plate of basal fold; outer median plate is internally branch connected to inner median plate, outwardly connected to median vein and cubital vein. Median plate plays an important role in the folding of wings.

\subsection{Wing movements}

Wing movements are divided into two types, namely folding and flying.

\subsection{Dipterization and wing chain}

3.3.1 The flight of dipterization insects mainly takes the alternative contraction of musculus tergi longitudinalis and dorsoventral muscles in wing chest as power, and musculus tergi longitudinalis of front and back chest both grow on the suspensorium of mesothorax posttergite. If two pairs of wings are all applied in the flight, it is possible that the simultaneous contraction of musculus tergi longitudinalis of middle and back chest affects the same suspensorium and thus imposes an adverse effect of tension cancellation. In theory, only one pair of wings loses their flying ability, and the other pair of wings would exert their flight performance.

\subsubsection{Wing chain}

In several insects that both wings could be used in the flight, the front and back wings is connected by several coupling apparatuses or claustrums in order to make them cooperate and coordinate with each other.

\section{Research Significance}

Wing is one of the most important organs of insects, and due to wings, insects adapt to different environmental conditions in the world. Insect wings would change along with the affection imposed by time and environment 
factors, and these changes might be closely associated with the genetic material variation and environmental condition influence. Sometimes shape and texture variation would usually cause the transformation of wing function. For example, wing depression and disappearance are restricted by many condition factors. Wings sometimes would depress due to ecological factors, for example, the ratio of no-wings, short-wings and wing depression in insects obviously increased; As far as carabid, darkling beetles, weevils and so on in the desert are concerned, their forewings exist and their underwings depress and disappear; Forewings in some species heal and underwings depress or are very underdeveloped, without flying abilities, and especially for the species in the desert, the healed elytra changes into a multi-functional storage room, namely sheath fossa. It has the effects of alleviating heat and preserving water in order to survive in the condition of desert and semi-desert. Wing texture change is mainly designed to protect the body of insects, and from half compound wings or semi-wing to compound wings, from compound wings to elytra, protective effects of membranous wings gradually increased, which might be the reason that insects of Coleoptera became the most prosperous. Insect wing shape variation was related to the protection of body, and in some species it was associated with mimicry. For example, double wings of several dead leaf butterfly were similar to leaves, and played a cardinal role in avoiding enemies; tails of many insect underwings in Lepidoptera had protuberances with various lengths, and these protuberances were of important significance to protecting body, especially for head (Sun, 1987).

The mystery of insect flight depends on its wing. Wings became one of the lightest structures by natural selection. The above part of wings was bent while below part was straight, and thus air flow above wing had a longer distance and faster speed than that below wings. Joint formed in wing base was connected with body, could flick upwardly and downwardly, and turn with the frequency ranged from 20 to 600times/s (Xiao, 2001, PP. 38-39). Compared to monotonous aircraft flying, insect wings could rotate in different directions at different time, and accomplished all kinds of highly difficult movements such as circling, diving, flashbacking and so on. Wing activities depended on a comprehensive manipulation of the chest muscles. Direct flight muscles were connected with wing base, and could manipulate the tilting, folding, rotating and upward and downward flapping of wings. Indirect flight muscles contained musculus tergi longitudinalis and dorsoventral muscles. The two kinds of muscles were attached to the inner wall of chest exoskeleton, and made wings flick upwardly and downwardly by changing the shape of chest (Zhang, 2003, P.12). As seen from the muscle size, this was the recognized most powerful muscle in nature. Over the centuries, zoologists, bionics scientists, space experts had continuously studied the insect trick flight. However, this issue has not been clearly elucidated so far. Insects applied unconsciously and simultaneously several space technologies, such as vortex production, delay devices, rotational circulation and coda capture, etc.; these techniques could be used for the research and development of micro air vehicle, for environmental monitoring and planetary exploration. Some scientists have reported in the top magazine of Nature that they created a special "wind tunnel", trained vanessa atalanta to fly in Atlantic, and blew a plume of smoke in order to show the vortex generated by the interaction between air and wings. Vortex was photographed by high-speed digital camera for analysis. The conclusion was that vibration manner of wing in flight was not casual, but conducted by subtle aerodynamic mechanisms. They could indentify six kinds of vibration manners. Butterflies changed their wing vibration manners in flight, which were as nature as running and galloping horses changed their running manners. It was anticipated that micro air vehicle simulating insects would come out soon. Even it seems to be a tiny wing, and it was worthwhile for us to learn from. Taken together, insect wings indeed had considerable mysteries for us to take further investigation.

\section{References}

Cai, W.Z. (2001). General Entomology. Beijing: China Agricultural University Press.

Sun Y., Wang, M.F., \& Xue, W.Q. (2004). Insect wings. Biological Bulletin, 39 (11).

Sun, Y.Y. (1987). Principles of Animal Ecology. Beijing: Beijing Normal University Press.

Xiao, Z. (2001). Secrets of insect flying. World Science, 11:38-39.

Zhang, Z.H. (2003). Animal movement. Biology Bulletin, 38 (10): 12. 


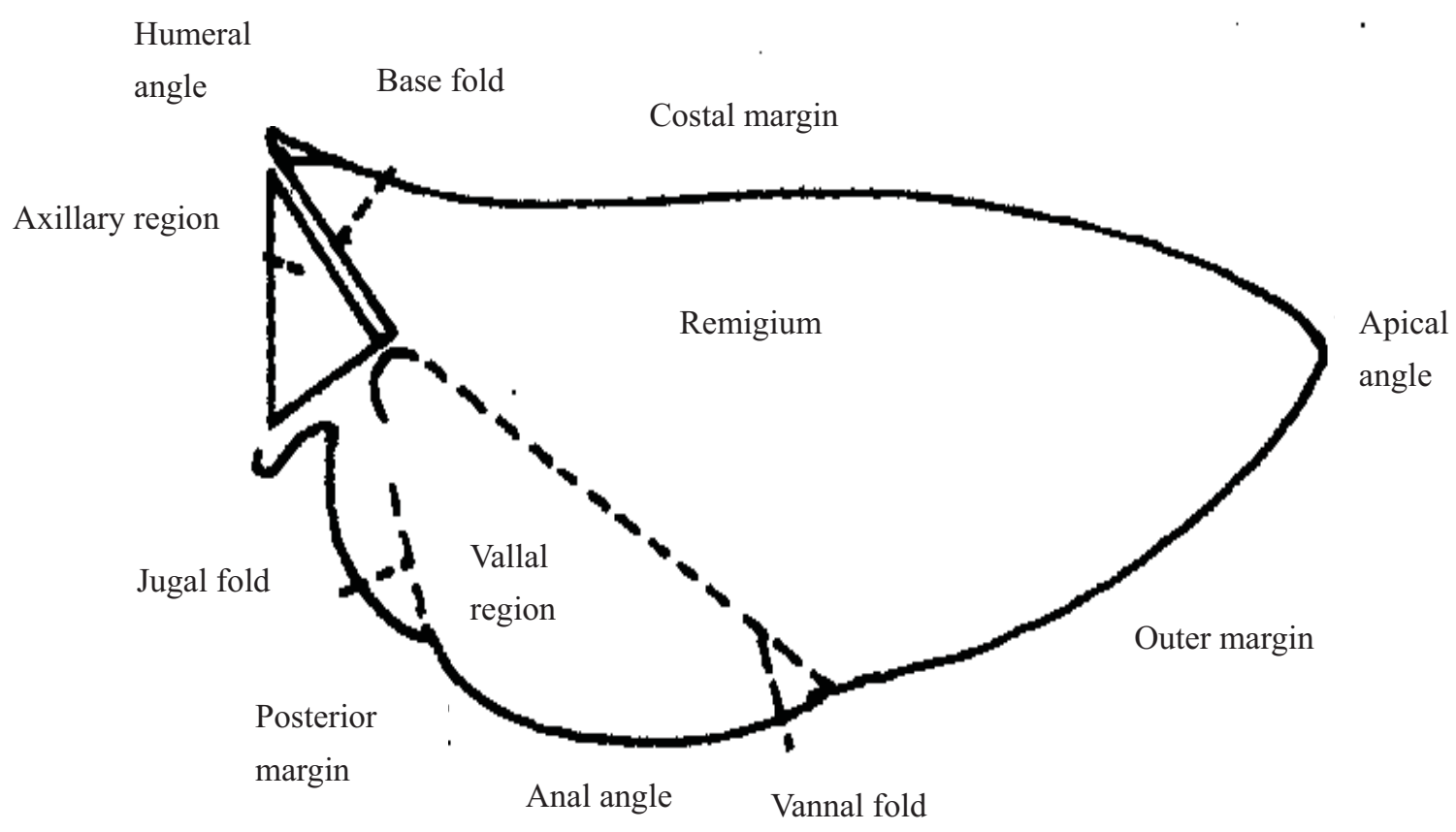

Figure 1. Basic structure of wings (Sun, 2004, P.11) 